\title{
CommentaRY
}

\section{Longitudinal rotating pathology elective: Reflections on a pilot project in pathology at Dalhousie}

Aleksandra Kajetanowicz,' Patrick Holland,' Carley Bekkers,' Deji Ologbenla,' Ashley Stueck, ${ }^{2}$ Thomas Arnason ${ }^{2}$

I. Class of 2020, Faculty of Medicine, Dalhousie University, Halifax, NS, Canada

2. Department of Pathology, QEIl Health Sciences Centre, Dalhousie University, Halifax, NS, Canada

\section{Abstract}

A new half-year longitudinal rotating pathology elective was offered to second-year Dalhousie medical students. The elective ran from January 2018 to April 2018 and again from January 2019 to April 2019. From both the student and preceptor perspective, the rotating elective provided a well-rounded experience in pathology, and is an excellent student introduction to residency programs and career options.

\section{Background}

First and second year medical students at Dalhousie University participate in clinical electives for a half-day per week as part of the pre-clerkship curriculum, with the goals of exploring an area of interest, gaining exposure to the clinical work environment, seeking out career options, and identifying potential clinician mentors. First year (Med1) students participate in one half-year elective, while second year (Med2) students have the option of a single full-year elective, or two half-year electives. Students typically organize these electives independently by approaching a physician or other healthcare professional as a preceptor with a specific proposal. In Med1, there is an opportunity in the Dalhousie electives catalogue to participate in an organized "rotating elective" where students are placed with clinicians in a variety of specialties. In past years the rotating elective has included Psychiatry, Family Medicine, Surgery, Internal Medicine, Pediatrics, and Obstetrics and Gynecology, each for two weeks at a time.

In 2018, two Halifax-based pathologists, Dr. Thomas Arnason and Dr. Ashley Stueck, organized a new half-year rotating elective for Med2 students based in Halifax, focused entirely on pathology and laboratory medicine. The elective was structured with a schedule where participating students spent their half-day with a different pathology and laboratory medicine preceptor each week. Preceptors included hematopathologists, microbiologists, clinical chemists, a neuropathologist, and anatomical pathologists with subspecialty interest in the gastrointestinal tract, liver, skin, breast, gynecologic tract, heart, lungs, and forensics. Teaching sites included pediatric and adult hospitals, as well as the provincial medical examiner's office. The goal was to increase student exposure to pathology before clerkship and to allow students to explore the broad range of residency and career options within pathology. Two second year medical students participated in 2018 and three in 2019.

Structured "rotating electives" are uncommon in the pre-clerkship years at Dalhousie, and this "rotating pathology elective" appears to be unique in Canada. We asked the students and lead preceptors to reflect on the experience and describe the challenges, opportunities, and impact of this type of structure for a second-year medical school elective. The student and preceptor perspective based on this experience might be helpful in planning similar rotating electives in other specialties at Dalhousie, or similar pathology electives outside of Halifax.

\section{The student perspective \\ What personal or professional growth did you experience?}

Patrick Holland noted a new appreciation for the breadth, volume, and importance of the work done in the laboratory behind the scenes: "When you send off a sample and a requisition, you don't really ever think about the amount of processing and evaluation that goes into that tissue, and all the people who are involved in that test, before you get the end result." Deji Ologbenla experienced a different dimension of patient care and observed how sample accession personnel, laboratory technologists, biochemists, pathologists and many other professionals all contribute to healthcare delivery without any patient contact. "Though pathologists and laboratory personnel do not have direct contact with patients, their work impacts patient care as they often come up with diagnostic as well as prognostic findings that guide patient management."

\section{Did the elective narrow your career goals?}

Students felt that pathologist preceptors and residents were candid in discussing the reasons why they chose their career paths and the experiences that led them to where they are now. Pathologist mentors discussed the job forecast within the lab medicine subspecialties and work-life balance in residency and as staff. For Carley Bekkers, who started the elective with a potential interest in a pathology career, the elective helped solidify that choice: "During the elective, I had 
the opportunity to discuss what a career in Pathology is like with staff and residents. Having these conversations helped me in coming to the realization that Pathology fits with what I am looking for in terms of a lifestyle and day-to-day job”. In contrast to other electives, students felt that this elective offered exposure to a greater variety of professional perspectives and opinions. Carley Bekkers noted: "Before the elective, I was specifically interested in forensic pathology, but I was pleasantly surprised at how much I enjoyed the other sub-specialties I was exposed to, particularly Pediatric Pathology, Neuropathology, and Hematopathology." Student participants felt that the elective helped them understand the difference between pathology residency specialties that they can apply to across Canada, and what careers are possible with those residencies.

Those considering pathology as a career felt that the timing of this elective was excellent, because it immediately precedes the core 3rd year clerkship. Aleksandra Kajetanowicz wanted a chance to learn more about pathology as an option for residency before the start of core clerkship: "After Med2, the next opportunity to spend time in pathology isn't until Med4 electives, and unfortunately students need to start applying for these electives mid-way through Med3. Without a pre-clerkship elective, you would be applying blindly to electives and hoping you enjoyed the work". Aleksandra found that during the pathology elective she got to understand the day-to-day work of a pathologist and found it appealing: "I was pleased to learn that I enjoyed the lab environment, and during the rotating elective I started to seriously consider pathology as a career choice." Now in clerkship, Aleksandra has been able to experience more blocks in clinical medicine and reflect back on the pathology elective: "It was difficult to admit to myself that patient interactions, although extremely rewarding, were also at times exhausting. I am very happy to have the experience of the rotating pathology elective to look back on and can now plan to do more electives in pathology after core clerkship. I hope to pursue a career in pathology and am looking forward to sorting out whether general, anatomical, or hematopathology is the best fit for me."

Patrick Holland started the elective curious about a career in pathology. He enjoyed the experience, but learned that is probably more suited to a career in family or internal medicine: "The pathologists were fantastic and I really enjoyed some of the daily activities like Gross Rounds, however I found that by the end of the 12 weeks I was missing seeing patients and talking to them about their issues." Deji Ologbenla has always been interested in pediatrics but also finds the idea of pathology fascinating, which led him to pursue the longitudinal pathology elective. He particularly appreciated his experience shadowing a pediatric pathologist: "As I have a very keen interest in pediatrics, it was exciting to spend time with a pediatric pathologist at the IWK who showed me that it was possible to be a pathologist and still channel your passion for helping children." The neuropathology component of the elective was also very well received and helped guide Deji towards exploring a neurology rotation in clerkship: "I really enjoyed the neuropathology week where we got to participate in a couple of brain autopsies. It involved a lot of dissection and understanding of neuroanatomy and this experience contributed to my decision to do a rotation in neurology at the Halifax infirmary during clerkship." Deji is hopeful his clerkship experience in Med3 followed by a fully-fledged elective in pediatric pathology early in Med 4 will shed more light and help clarify his path post medical school.

Did the elective influence how you will practice in the future?

Participants felt that the elective would influence their future practice regardless of whether or not they ultimately pursue a career in pathology. Carley Bekkers noted that her experience learning about the laboratory test cycle will help her approach to clinical care and reduce her risk of errors in clerkship and residency: "In rotating through clinical pathology labs, we discussed pre-analytical, analytical and post-analytical phases of lab testing and identified where errors in these phases could occur. I found this to be particularly valuable as no matter what specialty medical students pursue, having knowledge of how sample testing is done and how medical students, residents, and staff clinicians can reduce pre- and post-analytical errors is critically important to providing safe care for our patients". Deji Ologbenla suspects that the experience in the lab will encourage him to form collaborative relationships with pathologists and he hopes that he can become involved in the fast growing field of molecular medicine in his future practice: "Whichever path I take, I will form collaborative relationships with pathologists and try as much as possible to tap into the breadth of knowledge they possess. I also think molecular pathology is the future in diagnostic and prognostic medicine and will find ways to incorporate it into my practice."

\section{What challenges does the elective present to participating students?}

While the multiple preceptor / multiple subspecialty format provided a wide breadth of experiences, it created challenges in terms of building skills and assessing progress from week to week. Carley Bekkers noted that when you switch preceptors each week, "it does not necessarily provide the opportunity to work on new skills acquired, for example, recognizing and 
recalling the histological features of a specific tissue or organ. Having a limited time with each preceptor makes it challenging to assess the progress you make in the elective." Another downside to the multiple preceptor format of the elective is that there was less opportunity to build a strong relationship with an individual preceptor. However, this was mitigated by working three times with Drs. Stueck and Arnason at the beginning, mid-point, and end of the elective for evaluations and feedback. Deji thought he sometimes struggled with orienting himself to surgical specimens and the rationale for deciding to section them in a specific way for histology: "It was sometimes difficult to wrap my head around why a transverse cut was needed somewhere, and a longitudinal cut elsewhere as I got myself oriented to specimens during gross rounds". To mitigate this, we have suggested an orientation to gross pathology with a resident to help students in the elective understand the basics of gross pathology.

\section{The preceptor perspective}

How did preceptors respond to teaching second year students?

All of the preceptors had previously taught medical students, but the majority of the medical student electives in the pathology department are fourth year MD program electives. Pathology electives prior to fourth year are less common, and pathology is not part of the core clerkship curriculum. Preceptors responded to the concept of the elective very positively and it was relatively easy to recruit the group of 14 preceptors each year. According to Dr. Arnason "I thought the elective was a good team building exercise for the department, as we had preceptors participate from all different disciplines". Dr. Stueck noted "The preceptors frequently remarked that this elective presented a valuable and unique method of interaction with Med2 students and felt it would be favourable for recruitment to the pathology and laboratory medicine specialties."

\section{Any approaches to teaching which were more or less} effective?

Dr. Arnason's impression was that students seem to be more interested in participating in the pathologists' actual daily work rather than review teaching cases: "I think there is a temptation in teaching anatomical pathology to stop doing your work and start teaching a lesson in basic histology with an archived set of histology slides. However, my experience is that the junior medical students prefer to observe me doing my actual anatomical pathology cases for the day, with pauses to explain the clinical question on the requisition, the microscopy, and how I will write up the report for the case. Similarly, they don't mind at all if we are interrupted to do a frozen section. I think that this type of elective should place more emphasis on teaching medical students what it is like to have the job of a pathologist, rather than focusing on basic histology and microscopy skills." Dr. Stueck concurred: "For small group sessions, particularly when only a single interaction with the students is planned, pathologists tend to concentrate on archival teaching material that highlights histology of either very common or extraordinary cases. This approach may be better suited to pathology residents. For medical students, discussion of challenges and interpretation of current cases, including pre-analytic factors such as what was sampled and why and what medium the tissue was placed in and for what duration, and analytic factors such as gross features and how the specimen was grossed, seem to be more relevant and of interest."

In anatomical pathology, a focus on gross pathology and autopsy seemed to work well. Dr. Stueck observed: "The inclusion of autopsy pathology has been well-received and is well-timed for the Med2 students pre-clerkship. It is important for the students to understand what an autopsy entails, the choices available to the patient's family, and the timeline of the procedure, particularly as the students will likely be involved in speaking to families about, and consenting for, autopsy during clerkship." Dr Arnason added: "I think the Med2 students tend to have strong skills in anatomy and can recognize that an abnormality is present in gross specimens, so autopsies, brain-cutting, and gross rounds are easy for them to understand and learn from. I think histopathology has a steeper learning curve, but it's important they get that experience to see if the job is right for them".

\section{What challenges does the elective present to the organizers and preceptors?}

The main challenge from the faculty point of view was the complexity of developing and coordinating a schedule with multiple faculty preceptors that matches student availability - according to Dr. Arnason, "organizing this requires a lot of emails, phone calls, and hallway conversations." The elective coordinators knew that student evaluations would be a concern, they ensured that these evaluations would be completed by scheduling the students with two elective coordinators (Drs. Stueck and Arnason) at the beginning, midpoint, and end of rotation. Soliciting feedback from faculty about the students was relatively simple, mainly through a few short e-mails and conversations. While there was useful verbal feedback about the program and faculty from the students, it seemed unreasonable to ask for written individual preceptor evaluations for such a short experience with each preceptor. Lack of 
a written faculty evaluation could be perceived as a weakness of the program by some faculty. To help make up for this, at the end of the rotation, Dr. Arnason sent written thank you letters to all participating faculty.

As most preceptors had a single experience with the students, Dr. Stueck notes that "It is somewhat challenging for preceptors to form a relationship with the students and they lack the ability to observe skill and knowledge acquisition and application over time." Again, this is somewhat mitigated by recurrent exposure to the two main preceptors (Drs. Stueck and Arnason). This could be improved by repeat exposure to more preceptors, but the downside would be reduced variety. Dr. Stueck thinks "We must keep in mind the goal of this elective, which is broad exposure to pathology and laboratory medicine, rather than specific skill acquisition, and communicate this well to our preceptors and students before and during the elective."

Any suggestions for pathology preceptors looking to establish something similar at other institutions?

According to Dr. Arnason, one of the keys to the success of this type of elective is a department-wide, team-based approach to teaching, in order to provide an elective that is representative of all disciplines in lab medicine: "We used to do one-on-one electives with a single preceptor for 14 weeks which is a sometimes onerously large time commitment for individual pathologist preceptors and offers the student a relatively limited range of experiences in highly subspecialized academic centers. Having a team of preceptors in different fields of lab medicine improves the diversity of the student experience and it lightens the workload for each individual pathologist involved."

\section{Discussion}

In the Dalhousie medical school curriculum, the main exposure to pathology is in the first and second year through lectures, laboratories, and tutorial cases. There is no option to do a pathology rotation in the third-year core year of clerkship. This is typical of most Canadian medical schools. Without a core clerkship rotation and without well-established elective opportunities for medical students, there is a theoretical risk that that medical students with an aptitude and interest in pathology may fail to pursue residency and a career in the specialty due to a lack of exposure to the clinical practice and a lack of mentorship.

In the same year that this faculty-driven, rotating elective in pathology began, there was a new medical student-driven elective at the end of Med2 which also included pathology. The elective, called "PREP" (Pre-clerkship Residency Exploration Program), is reviewed on the Dalhousie University website ${ }^{1}$. The PREP elective provided the 40 participating Med2 students with a brief clinical experience in 14 different medical specialties. In the two weeks, each student spent one day in pathology: a half day pathology preceptorship and a half day pathology skills lab. The PREP elective provides a larger number of students with a much shorter duration clinical experience in pathology. The experience was not standardized, and each small group of students were exposed to different preceptors from different laboratory medicine specialties. While the PREP elective might be enough to spark an interest or rule out pathology as a career choice, the longitudinal rotating elective provides a more well-rounded experience and may better assist students with a career decision, particularly if they are already seriously considering pathology.

Increasing medical student interest and exposure to laboratory medicine careers is a long-term goal for the longitudinal rotating and PREP electives. Whether these electives are influential will take years to evaluate, and may be difficult to assess, since so many variables affect career decisions. However, we think there is value in the experience regardless of recruitment outcomes. The elective provides a hands-on opportunity to learn about laboratory tests, which is useful regardless of whether or not participating students eventually pursue or rule out pathology as a career choice.

\section{References}

1. Conrad, K. Student run program showcases careers in different medical specialties [Internet]. 2018 Aug 27 [cited 2020 May 27]. In: Dalhousie University Faculty of Medicine News. Available from: https://medicine.dal.ca/news/2018/08/27/student_run program_showcases_careers_in_different_medical_specialties. html 